\title{
TRCCIT: Tunable Reliability with Congestion Control for Information Transport in Wireless Sensor Networks
}

\author{
Faisal Karim Shaikh, Abdelmajid Khelil, Azad Ali and Neeraj Suri \\ Technische Universität Darmstadt, Germany \\ \{fkarim|khelil|azad|suri\}@cs.tu-darmstadt.de
}

\begin{abstract}
A core functionality of Wireless Sensor Networks (WSNs) is to transport information from the network to the application/user. The evolvable application reliability requirements and the fluctuating perturbations lead to continuous deviation between the attained and desired reliability. Using an existing approach that guarantees a highest reliability is not appropriate for WSN as this over-provisioning wastes the most valuable resources, e.g., energy. In this paper, we present a new approach called as Tunable Reliability with Congestion Control for Information Transport (TRCCIT) in WSN. To provide probabilistically guaranteed tunable reliability TRCCIT implements localized techniques such as probabilistic adaptive retransmissions, hybrid acknowledgment and retransmission timer management. TRCCIT pro-actively alleviates the network congestion by opportunistically transporting the information on multiple paths. TRCCIT fulfills application reliability requirements in a localized way, which is desirable for scalability and adaptability to large scale WSNs. Simulation results show that TRCCIT provides tunable reliability and efficiently mitigates the congestion.
\end{abstract}

\section{INTRODUCTION}

Wireless Sensor Networks (WSNs) constitute a rapidly growing research area, covering both a wide variety of devices and applications. Typically, a WSN comprises number of sensor nodes possessing limited processing and power capabilities, often communicating over unreliable and low bandwidth radio links [1]. Empirically, the core operation of a WSN is to transport the information of interest from the network to the application/user via a gateway node termed as sink. The application requirements impose consequent reliability requirements for the base information transport in a WSN.

Being an ad-hoc and volatile environment, the WSN is obviously subject to a wide range of perturbations that lead to deviation between the attained and desired reliability complicating the design of transport protocols in WSN. There has been intensive research to design suitable transport protocols for WSN. To achieve reliability, existing information transport protocols exploit temporal redundancy (e.g., retransmissions [2], erasure codes [3]), spatial redundancy (e.g., number of sources [4] or paths [5]) or some combinations of them [6]. These works mitigate perturbations to some extent, however, are not able to cope with evolving network conditions as they are designed for specific classes of applications. Our comparative study [7] has additionally confirmed that the current approaches perform well only for carefully selected deployment scenarios. Furthermore, the existing protocols are not designed to explicitly consider the varied or evolvable application requirements as their main design driver is to efficiently maximize the attained reliability. However, it is obvious that a WSN runs different applications (network management, event detection, event perimeter tracking etc.) that require different information types with varied transport reliability requirements. It is also expected that the same WSN application may tune its requirements over time. The existing approaches over utilize the precious network resources even when the application does not require more reliability. Therefore a tunable information transport reliability is required which fulfills the application requirements and also adapts according to network conditions.
A particular perturbation for information transport is the occurrence of network congestion. Generally, congestion is caused by a high network load, which typically manifests when bursty information has been generated by sensor nodes and sent to the sink. Sensor nodes experience congestion due to a high incoming information rate compared to the maximum possible outgoing rate to the next hop node. To mitigate this problem many back pressure schemes have been proposed in the literature, which reduces the incoming information rate [8], [9]. It is not always desirable to throttle the information of interest, since it can effect the desired reliability of the application. Therefore, some recent works suggest measures to increase the outgoing information rate either by increasing the resources around the sensor nodes [10], [11] or using multiple paths [12]. These works mitigate congestion reactively which leads to the dropping of the information. Also none of these works considers the application reliability requirements. Our approach pro-actively mitigates congestion and provides application specific reliability.

To the best of our knowledge we are the first to consider the tunable reliability of information transport combined with congestion control in face of evolving network conditions and application requirements. In this paper, we present Tunable Reliability with Congestion Control for Information Transport (TRCCIT) that exploits and adapts between the temporal (adaptive retransmissions) and spatial (multiple paths) redundancies inside the network. Specifically, we enhance our previous work Adaptive Reliable Information Transport (AReIT) [13] by including hybrid acknowledgement (HACK) and local timer management to ensure tunable reliability and provide a comprehensive congestion control mechanism. The new aspects of TRCCIT are four fold. First, we identified the problem of implicit acknowledgement (IACK) loss in AReIT which resulted in HACK for TRCCIT. Second, AReIT retransmission timers are managed at source node by observing the buffer status of next hop nodes, which requires additional information exchange. In contrast TRCCIT observes only local buffers, thus, avoiding this overhead. Third, TRCCIT provides statistical bounds for information transport and through simulations we show that the information transport is always statistically bounded. Finally, TRCCIT provides congestion control on the fly by adapting pro-actively between single and multiple paths. Paper Contributions: Based on the given background this paper makes the following contributions.

- We develop TRCCIT approach to ensure the desired reliability despite evolving application requirements and network conditions.

- We develop localized mechanisms such as HACK and retransmission timer to achieve tunable reliability of information transport.

- We develop a simple yet generic congestion control mechanism which pro-actively detects and mitigates congestion in order to maintain the desired reliability requirements. 
The rest of the paper is organized as follows. Section II presents the related work followed by Section III detailing the system, perturbation, information and reliability models. Section IV presents the TRCCIT approach. We evaluate TRCCIT in Section V. Our conclusions and directions for future work appear in Section VI.

\section{RELATED WORK}

We classify the related work into three areas: (1) aims at increasing reliability in non congested WSNs, (2) focuses only a specific perturbation, i.e., congestion and (3) deals with both.

(1) Reliability: In this area, the major focus is on increasing the information transport reliability as much as possible. In order to increase reliability on a single path to the sink, different acknowledgement (ACK) schemes are widely adopted. [14] sends the sequence of packets to the next hop with explicit acknowledgement (EACK) to ensure reliability. Reliable Multi-Segment Transport [2] and Asymmetric Reliable Transport [15] utilizes timer driven retransmissions for loss detection and notification. Above solutions provide always higher reliability than required. On the other hand our approach provides tunable reliability according to application requirements. Distributed Transport for Sensor Networks (DTSN) [16] and Sensor Transmission Control Protocol [17] provide differentiated reliability using end-toend retransmissions. DTSN beside retransmissions uses forward error codes (FEC) to enhance reliability. End-to-end retransmissions do not respond quickly in face of perturbations, thus, we adopted hop-by-hop retransmission strategy. On the other hand FEC requires a high level of computation, thus, limiting its practicality for WSNs. For high information rates, Reliable Bursty Convergecast (RBC) [6] provides a reliability design based on a windowless block ACK and IACK along with fixed number of retransmissions. RBC lacks tunability and always provides high reliability.

Another approach to increase the reliability is to utilize multiple paths. In [18] MMSPEED protocol is proposed for probabilistic quality of service guarantees in WSNs supported by multipath forwarding. MMSPEED comprises two modules, i.e., reliability and timeliness. The messages are forwarded according to their reliability cum timeliness requirements. Most of multipath approaches [19], [20], [21], [22], [23] proactively maintain multiple paths from each sensor node to the sink with the primary goal of load balancing and enhancement of the network lifetime. Our focus is on reliability and the TRCCIT approach adapts between single path and multiple paths based on the information rate flowing across sensor nodes and avoids creating and maintaining multiple paths which are costly in terms of number of transmissions.

(2) Congestion Control: In this area, the major focus is on the detection and mitigation of congestion. The existing approaches [8], [9], [24], [25], [26] limit information rate to mitigate the congestion which may not be desired for fulfilling the application requirements. All these works consider that if the path is congested, the whole network is congested which may not be the case. These approaches require feedback from the sensor nodes, which results in an extra network overhead. Moreover, none of these approaches focuses on the tunable reliability. Reducing congestion with multipath routing has been addressed by some recent works [10], [11], [12]. Our approach provides tunable reliability and mitigates congestion proactively before it actually happens by monitoring the incoming and outgoing information across sensor nodes.

(3) Reliability with Congestion Control: There are few works in this area which consider both reliability and congestion control. The Event to Sink Reliable Transport (ESRT) protocol [4] achieves reliability by adjusting the reporting rate of sensor nodes depending on the current network load. Upon congestion detection sensor nodes inform the sink for appropriate reaction. This approach may not reflect the current status of the network appropriately thus resulting in wastage of network resources. Our work provides tunable reliability at the hop level, whereas ESRT provides best effort end-to-end level reliability which is difficult to maintain, especially in large scale WSN. Similarly, [27] also provides an end-to-end best effort reliability. Authors in [28], consider multiple sinks and two classes of information reliability. Each sink receives either low or high priority information based on its role. The proposed algorithms in [28] degrades the low priority data by suppressing them to forward high priority data. Our approach provides tunable reliability compared to [28] which provides only two levels. Also we provide equal opportunity for all information to transport towards the single sink, however under different reliability guarantees.

As also emphasized by recent surveys [29], [30], there is a lack of an integrated approach that provides tunable reliability for information transport, combined with congestion control in WSN. Our TRCCIT approach fills this gap and provides tunable reliability along with congestion control in an efficient manner.

\section{Models \& ClASSIFICATION}

We first present a simple yet comprehensive system and perturbation model to capture generic WSN properties. Next, we discuss the information and reliability models.

\section{A. System Model}

We consider a WSN having $N$ sensor nodes numbered [0.. $N-1]$ with node 0 termed as sink. Typically, each node is equipped with one or more sensing devices, short range transceivers for communication, limited processing, less memory and restricted energy capabilities. We consider a sink to be adequate in power, more memory and higher processing capabilities as compared to the sensor nodes. We assume that all nodes are static in nature including the sink and are placed in a finite size area. Sensor nodes communicate with each other via bi-directional multi-hop wireless links employing a Carrier Sense Multiple Access/Collision Avoidance (CSMA/CA) based Medium Access Control (MAC) protocol. Each sensor node maintains two buffers, i.e., input buffer $\left(q_{i}\right)$ and output buffer $\left(q_{o}\right)$. For any two Nodes $X$ and $Y$ we define their link quality $L Q=p_{(X, Y)} \cdot p_{(Y, X)}$, where $p_{(X, Y)}$ and $p_{(Y, X)}$ indicate the probability that a message sent by Node $X$ is received correctly by Node $Y$ and viceversa. $X, Y$ are defined to be neighbors, if $L Q \neq 0$. This implies that IACK can be used in our model, since neighbors can always hear each other. Let the sequence of hops $\left(X, H_{1}\right),\left(H_{1}, H_{2}\right) \cdots\left(H_{f}, 0\right)$ is a path Path $_{i}$ from Node $X$ to the sink. All nodes know the hop distance $h(X)$ towards the sink, i.e., each node knows how far it is from the sink in terms of number of hops. Also each sensor node has knowledge of its 1-hop neighbors. Based on hop distances the neighbors of a node can be classified as upstream neighbors, downstream neighbors and equal neighbors. To this end we denote the set $N_{u}=\{Y:\{X, Y\} \in$ $\mathbf{N} \wedge h(Y)=h(X)+1\}$ as the upstream neighbors of a Node $X$, the set $N_{d}=\{Y:\{X, Y\} \in \mathbf{N} \wedge h(Y)=h(X)-1\}$ as its downstream neighbors, and the set $N_{e}=\{Y:\{X, Y\} \in \mathbf{N} \wedge h(Y)=h(X)\}$ as its equal neighbors, i.e., neighbors at same hop distance.

\section{B. Perturbation Model}

Information transport essentially requires the identification and classification of the relevant node and communication perturbations that can occur in the considered system model. We mainly emphasize the temporal evolvability of these perturbations, which hinders in 
maintaining the desired level of application reliability. Cheap hardware, limited resources and severe environmental conditions lead to frequent failures in WSNs [31]. We classify the failures in WSN with respect to message loss due to both communication and node level failures.

Communication Level Failures: Communication disruptions constitute the most frequent failures hindering information transport in WSN. High bit error rates of wireless links, collisions and contention constitute the major causes of message loss.

Node Level Failures: At node level message loss is caused by dropping messages from full buffers. In this work, we neither consider message loss due to node failure (e.g., HW or SW) nor deliberate failures (e.g., intrusions).

\section{Information Model}

We refer to an information entity by a raw or an aggregated sensor data that is required by the application. Information entities can be generated centrally on a single node (e.g., an aggregating node) or in a distributed manner by some nodes. In the later case we say the information entity is replicated. The information entities can further be grouped/composed for a higher semantic such as grouping the location of the nodes that detected the same event and define a new information, i.e., the event/region perimeter. Accordingly, we classify the information required by the applications into two broader classes: Atomic information and Composite information. Atomic information is composed of a single information entity whereas composite information is composed of more than one information entities. In case where atomic information is generated at many sensor nodes we refer to it as replicated atomic information. Composite information can be viewed as a set of atomic information (spatial composition) and if it can be centrally aggregated (temporal composition), then it is transformed into a single atomic information.

\section{Application Requirements \& Reliability Model}

Maximizing efficiency is a primary design goal of WSN due to limited energy resources, therefore, we have to reduce message overhead as maximum as possible. Thus, WSN should transport critical information with high reliability potentially using more transmissions, while transporting less critical information at a lower reliability using less transmissions. Furthermore, composite information has finegrained requirements on reliability, i.e., depending on the shape and size of phenomenon the application requirements are changing and may require all the nodes or a subset of nodes to report. This necessitate to provide $\mathrm{x} \%$ (probabilistically-guaranteed) reliable information transport instead of best effort or transporting all information entities. Based on the application requirements we define atomic information transport reliability as the degree of tolerating the information loss over time. For composite information it is defined as how much loss of information entities can be tolerated by the application without loosing the semantic of the composite information. We express $x \%$ by a probability with which the WSN transports information entity towards the sink.

In this work, we model a composite information as a set of independent atomic information to be transported. We assume that the source node of an atomic information knows probability (which takes into account the set of atomic information composing the composite information or the degree of an atomic information replication). In this work, we also assume that atomic information is realized through a single message and is generated by a single sensor node. Furthermore, we consider high information rates, i.e., bursty information to be transported to the sink. In such a case the information is atomic in nature and has same properties as discussed above except the information rate is high. Since composite information comprises a set of atomic information, it is easy to transport the composite information by extending the solution of transporting a single atomic information with the given probability.

\section{TRCCIT: THE PROPOSED SOLUTION}

We first give an overview of TRCCIT. Then, we show how it adaptively integrates and controls different temporal and spatial redundancy techniques to provide tunable reliability of information transport and mitigate congestion on the fly.

\section{A. TRCCIT Overview}

The main motivation behind the TRCCIT is to provide desired reliability of information transport despite evolving application requirements and network conditions, i.e., collisions and congestion. The TRCCIT tunes the temporal and adapts the spatial redundancy to provide tunable reliability of information transport. Here we focus on how TRCCIT integrates and adapts these parameters such that the desired application reliability $\left(R_{d}\right)$ is fulfilled. When the information rate is low and sensor nodes do not observe congestion, TRCCIT utilizes the default single path (SP) for transporting the information. In order to provide tunable reliability the TRCCIT exploits the temporal redundancies and tunes the number of retransmissions according to $R_{d}$ and network conditions. High information rate leads to the congestion inside the WSN [11] where the incoming information rate $\left(\xi_{i}\right)$ across a node is high compared to the outgoing information rate $\left(\xi_{o}\right)$. Here $\xi_{i}$ is defined as the number of incoming messages at the sensor node, either generated by the node itself or the forwarded messages and $\xi_{o}$ is defined as the number of outgoing messages. $\xi_{i}$ and $\xi_{o}$ can be measured at each Node $X$ on a message by message basis. Such burst of information usually creates a transient congestion inside the network, especially when the different information flows meet [10]. In order to alleviate congestion there should be some balance between $\xi_{i}$ and $\xi_{o}$. Once the information accumulates at the sensor node, which is an indication that the next hop along the path is not able to accept the information, it is required to take some measures. We aim at alleviating these transient congestions by quickly adapting the sensor node behavior by increasing $\xi_{o}$, i.e., dispersing the information to appropriate neighbor nodes such that the information transport is carried on multiple paths. It should be noted that by dispersing to neighbors may not result in completely disjoint paths rather it will provide braided paths. Furthermore, sometimes it may not be possible to send the information to any neighbor since the channel is busy. To avoid such situation we utilize simple methodology to randomly wait before transporting the information to next hop towards sink. Also further sophisticated methods can be utilized such as Request To Send/Clear To Send (RTS/CTS) and prioritized MAC protocols, e.g., [32], in order to provide fair chance for every node to access the channel.

\section{B. Tunable Reliability by TRCCIT}

Consider Node $X$ transporting information via Node $Y$ along a SP $P_{a t h}, h$ hops away from the sink. To ensure reliability across a hop $(X, Y)$ and to overcome node and communication level perturbations, i.e., message loss, more than one transmissions are required. Let $r$ be the number of transmissions required then the information transport reliability across a hop $R_{h_{d}}$ will be:

$$
R_{h_{d}}=1-\left(1-R_{h o p}\right)^{r}
$$


where $R_{h o p}$ is the reliability across a hop. Since $r$ is the total number of transmissions therefore number of retransmissions \# ret $=$ $r-1$.

For the $R_{d}$ imposed by the application and known number of hops from the sink, a sensor node can calculate the desired reliability requirement across a hop as:

$$
R_{h_{d}}=\left(R_{d}\right)^{1 / h(X)}
$$

Eq. (2) considers a uniform reliability requirement across the hops along the path. $R_{h_{d}}$ is the portion of the reliability requirement assigned to the hops by the source node along the path. For optimal allocation the source node must have a global knowledge, which is hard to achieve in WSN due to higher costs, i.e., number of transmissions. Therefore, Eq. (2) provides good tradeoff and relies only on local knowledge. If at any hop the reliability assignment is not optimal it will be compensated by adaptive number of transmissions as discussed below. The source node includes $R_{h_{d}}$ inside the message when it is transported to the next hop.

When a sensor node has an information to transport, it first decides whether to send the information to the next hop or suppress it in order to provide $R_{d}$. The decision is based on node's local network conditions and application requirements, i.e., $R_{h o p}$ and $R_{h_{d}}$ as follows:

$$
p_{s}= \begin{cases}R_{h_{d}} / R_{h o p}+\Delta_{t h} & \text { if } R_{h o p}>R_{h_{d}} \\ 1 & \text { if } R_{h o p} \leqq R_{h_{d}}\end{cases}
$$

where $0<\Delta_{t h} \ll 1$

When $R_{\text {hop }}>R_{h_{d}}$ the sensor node sends the information with probability $p_{s}=R_{h_{d}} / R_{\text {hop }}+\Delta_{t h}$ in order to maintain the required information transport reliability. This step ensures that the achieved information transport reliability is statistically bounded by $R_{d}$ and $R_{d}+\Delta_{t h}$. For the later case source node always sends the message to its next hop along the path. Once the decision of sending the information is taken by the sensor node it will calculate the maximum number of transmissions required to maintain the $R_{h_{d}}$ using Eq. (1) as follows:

$$
r=\left\lceil\frac{\log \left(1-\left(R_{h_{d}}\right)\right)}{\log \left(1-R_{\text {hop }}\right)}\right\rceil
$$

Note that at each hop along the SP nodes dynamically adapts $r$ to compensate the decisions made at previous hop to ensure the $R_{h_{d}}$. Since it is wasteful to send the $r$ transmissions if the next hop node have already received the information. To enhance this TRCCIT utilizes a hybrid acknowledgment scheme and local timer management to ensure $R_{d}$ across the path. Alg. 1 describes the complete procedure for TRCCIT to achieve tunable reliability.

1) Hybrid Acknowledgment Scheme: TRCCIT takes advantage of the broadcast nature of the WSN to receive IACK. A sender Node $X$ starts a retransmission timer after sending an information to the next hop Node $Y$ (Alg. (1):lines 18-22). If Node $X$ receives the IACK it will discard the retransmission timer and purge the information from the buffer else after timer expires it will retransmit the information to Node $Y$ (Alg. (1):lines 16-26). If Node $Y$ after receiving the information from Node $X$ decides to suppress the message to maintain the $R_{h_{d}}$ as discussed earlier (Eq. (3)), it will send EACK back to Node $X$ (Alg. (1):lines 28-31). Using this hybrid scheme saves the extra retransmissions carried out by Node $X$ in order to fulfill its reliability requirements since it did not hear the IACK due to suppression at Node $Y$. In order to avoid IACK loss, i.e., if the information is forwarded by the next hop and the source node was not able to receive it, Node $Y$ keeps the message for random

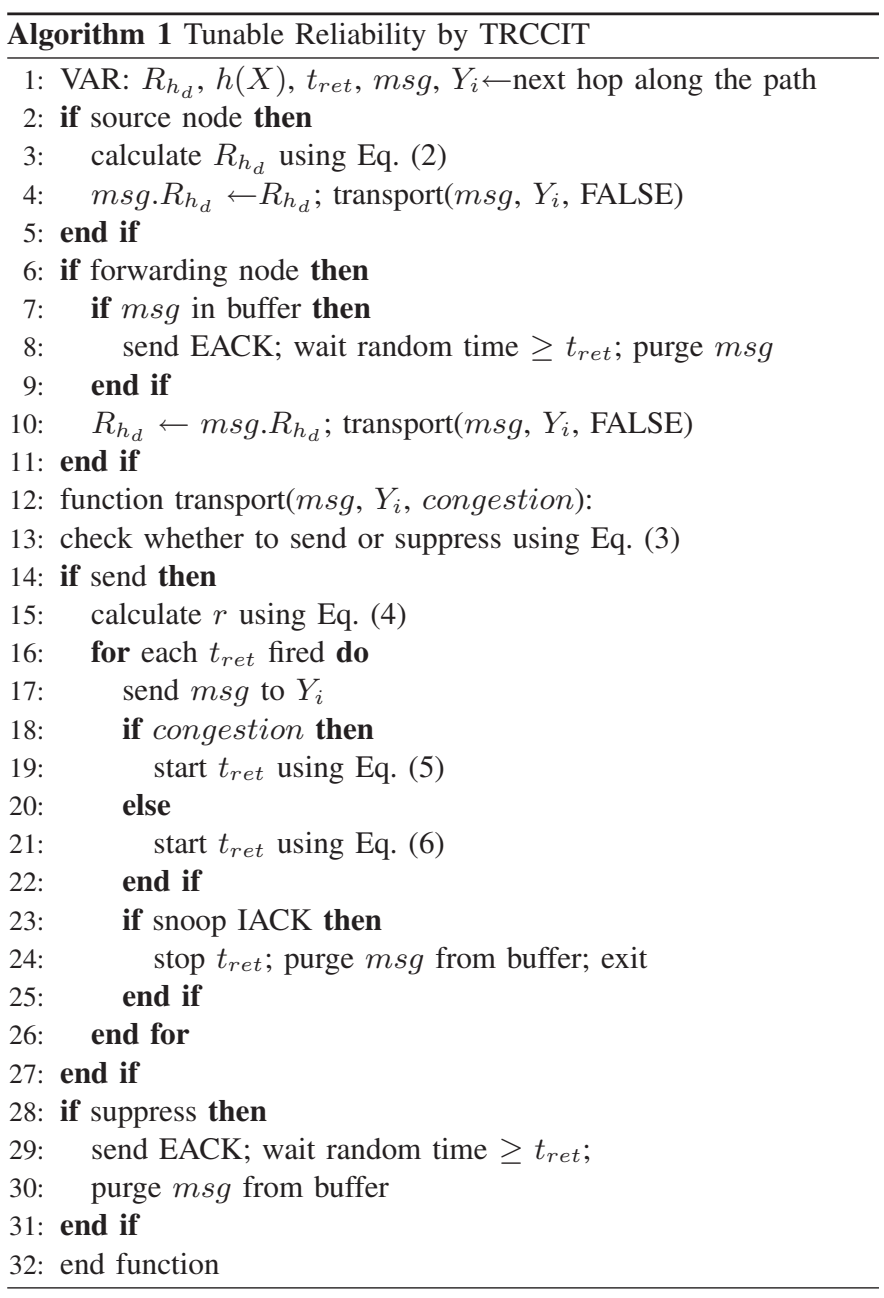

time $\geqq$ retransmit timer (as discussed in IV-B2) before purging it. If during this time Node $Y$ receive the message from Node $X$ it sends EACK back to Node $X$ (Alg. (1):lines 7-9).

2) Local Timer Management: Retransmission timers directly impact the number of transmissions. Large timeout values of timers tend to increase information transport delay, whereas small timeout values tend to cause unnecessary retransmissions. To provide reliable information transport, we design a simple and localized mechanism to manage the retransmission timers. Ideally, Node $X$ should adapt the retransmission timer according to the buffer status of next hop node. Since the number of messages in the next hop node keeps changing, the delay in forwarding a received message by Node $Y$ also keeps changing, which leads to varying delay in IACK. Since the overall network conditions are steady before congestion happens, we can assume similar conditions around the sensor nodes, i.e., similar $\xi_{i}$ and $\xi_{o}$. Based on this Node $X$ calculates the retransmission timer $\left(t_{r e t}\right)$ according to its $\xi_{o}$ and its buffer occupancy, i.e.,

$$
t_{r e t}=O\left(q_{i}\right) \cdot\left(t_{\xi_{o}}\right)
$$

where $O\left(q_{i}\right)$ is input buffer occupancy and $t_{\xi_{o}}$ is the time to send $\xi_{o}$ messages. This will reduce the overhead of getting the neighbor nodes buffer status. Once a node detects congestion due to high information rate as discussed in Section IV-C, it calculates the retransmission timer as follows:

$$
t_{r e t}=O\left(q_{i}\right) \cdot\left(t_{\xi_{o}}+4 t_{\xi_{o}}^{\prime}\right)
$$


where the deviation $t_{\xi_{o}}^{\prime}$ is utilized due to the fact that $t_{\xi_{o}}$ varies when information rate is high since it improves the calculation of retransmission timer [6].

\section{Congestion Control by TRCCIT}

In order to provide proactive congestion control each sensor node in the WSN monitors the local network conditions and the information rate across itself. TRCCIT provides more generic and simple method to precisely measure the information rate at each intermediate sensor node using $\xi_{i}$ and $\xi_{o}$. Alg. 2 describes the overall TRCCIT congestion control mechanism. Each sensor node keeps an exponentially weighted moving average (EWMA) $\xi_{i}(t)$ and $\xi_{o}(t)$ over a short time window $T$ of the number of messages that it is transporting (Alg. (2) line:4-5) where $\alpha$ is a weight-factor ranging between $0<\alpha<1$. The EWMA approach avoids the wrong node decisions due to sudden or abrupt changes. Based on this the transient congestion $\left(C_{t}\right)$ can be defined as

$$
C_{t}=\frac{\xi_{o}(t)}{\xi_{i}(t)}
$$

The ratio of $\xi_{o}(t)$ and $\xi_{i}(t)$ is an efficient indicator of transient congestion and is more robust then approaches like [4], [9], [24] where congestion is detected based on some threshold on buffer occupancy or complex mechanism of timer for incoming and servicing the messages. On the contrary TRCCIT only requires count of incoming and outgoing messages. If at time $t$ the ratio $\xi_{o}(t) / \xi_{i}(t)<1$, the sensor node detect the presence of congestion due to high information rate, i.e., $\xi_{i}$ is higher then $\xi_{o}$. On the other hand $\xi_{o}(t) / \xi_{i}(t)>1$ represent low information rate across the node and thus no congestion is observed (Alg. (2) line:6-10).

To this end, a set of $N_{d}, N_{e}$ as well as $N_{u}$ is maintained for each sensor node sorted in the order of highest hop reliability across neighbors. More specifically, each Node $X$ maintains the hop reliability $R_{h o p_{\left(X, Y_{i}\right)}}$ and hop distance to the sink $\left(h\left(Y_{i}\right)\right)$ to each of its one hop neighbor $Y_{i}$. Once Node $X$ detects the high information rate, it disperse the information to the set of neighbor nodes, i.e., $N_{d}, N_{e}$ and $N_{u}$, in round robin fashion (Alg. (2) line:1630). By this sensor nodes disperse the information such that the congestion will not accumulate on the single path. By dispersing information from the point of congestion to the neighbor nodes essentially provide the braided multiple paths. This braided effect is useful since information will converge to the optimal path as soon as it bypasses the congestion spot in the network. The sensor node which detects the congestion determines the neighbor nodes in $N_{d}$ and transports the next information in $q_{o}$ to it. Node $\mathrm{X}$ traverses the whole set and sends information to each neighbor one by one. If there are no more neighbors to chose from (i.e., $N_{d}=\emptyset$ ), the nodes from $N_{e}$ are selected to transport the information. In the worst case if both sets are empty, the information is transported to the neighbors in $N_{u}$. TRCCIT utilizes only the local knowledge to disperse the information to its neighbors. If Node $X$ selects the neighbor node in $N_{d}$ it will not change the reliability assigned by the source node, i.e., $R_{h_{d}}$ since the number of hops remains same for information to travel. If neighbor node is from set $N_{e}$ or $N_{u}$, Node $X$ recalculates the desired hop reliability as follows

$$
R_{h_{d}}^{\prime}=\left(\frac{R_{d}}{R_{h_{d}}}\right)^{1 / h\left(Y_{i}\right)}
$$

where $R_{h_{d}}$ is the previously calculated reliability available from the received message and $h\left(Y_{i}\right)$ is the hop distance of the neighbor. If Node $Y_{i}$ also experiences high information rate it will follow the same procedure and send the information to its neighbors. By doing so eventually the information bypasses the congestion area and starts flowing towards the sink.

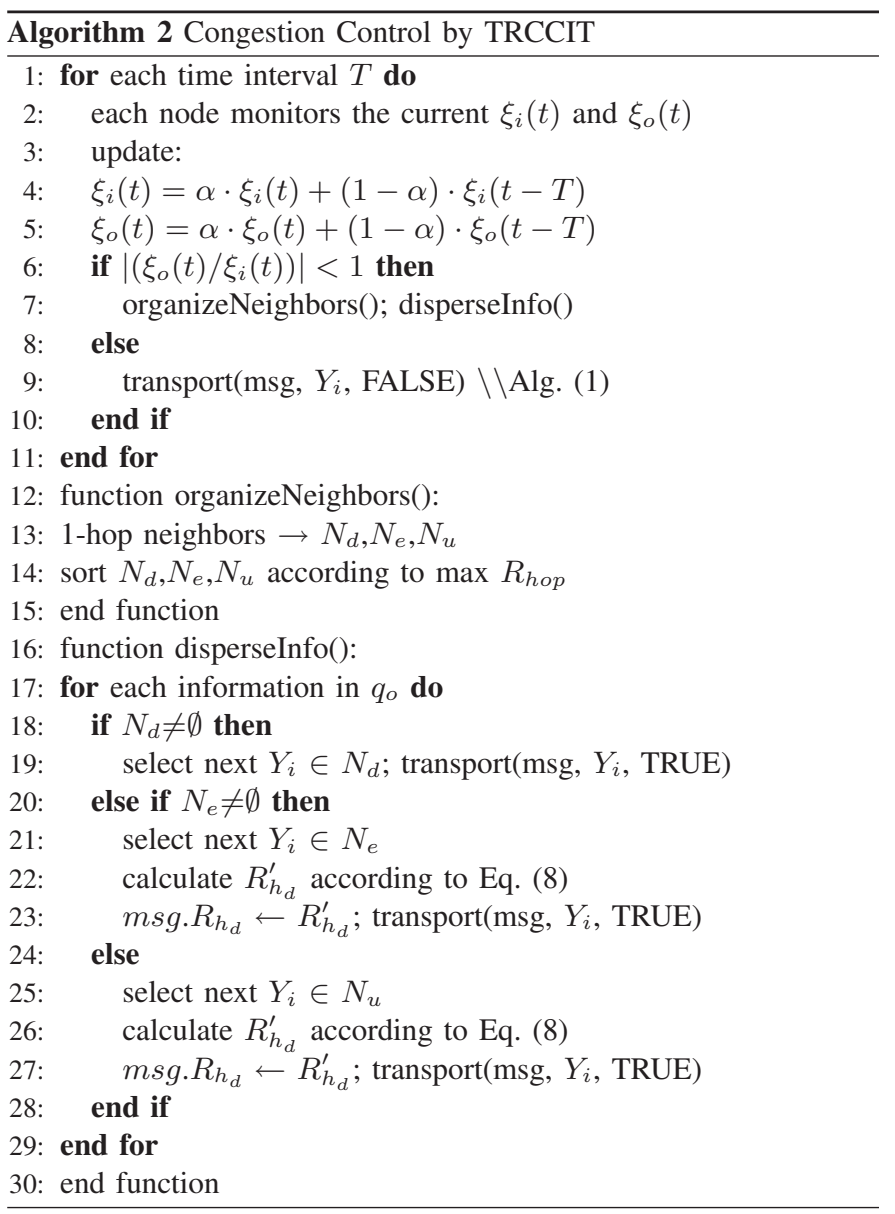

\section{TRCCIT Parameters}

TRCCIT rely on different parameters to be available by sensor nodes. In order to acquire hop count $h(X)$ the sensor node utilizes the underlying routing protocol. When the network initializes and the sink creates spanning tree, it includes a hop counter to beacon messages, which allows sensor nodes to acquire knowledge of $h(X)$, i.e., how far they are from the sink. The sink periodically sends beacon messages to all nodes such that a routing tree rooted at the sink is maintained. Furthermore, a change request for the desired application requirement $R_{d}$ can also be disseminated to the sensor nodes via routing layer, e.g., through piggy backing to beacon messages. It should be noted that in this work we emphasize on information transport from sensor nodes to the sink for ensuring tunable information transport reliability and not on reliable dissemination of application requirements to sensor nodes. To this end the sink can use existing reliable downstream dissemination strategies, e.g., [33], [15], [34] to distribute the $R_{d}$. In order to maintain $R_{h o p}$, a sensor node keeps track of the link quality between its neighboring nodes using EWMA approach. A sensor node keeps track of link quality in terms of bit error probability (BEP) between itself and its neighboring nodes upon reception of a message or when it snoops the channel for IACK. At a node, other local parameters such as $t_{\xi_{o}}$ and $t_{\xi_{o}}^{\prime}$ are calculated similarly using EWMA approach. 


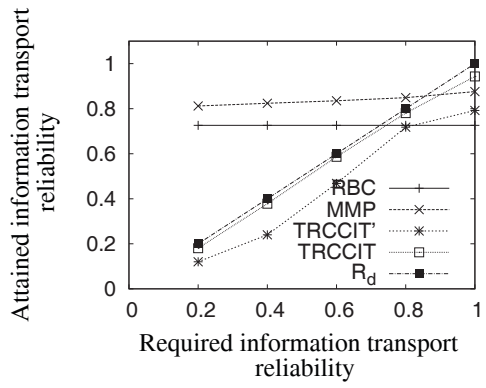

(a) Information transport reliability

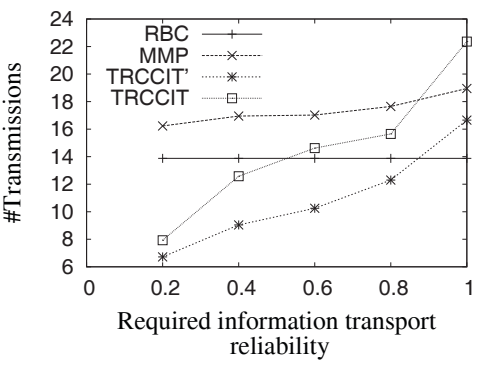

(b) Information transport efficiency

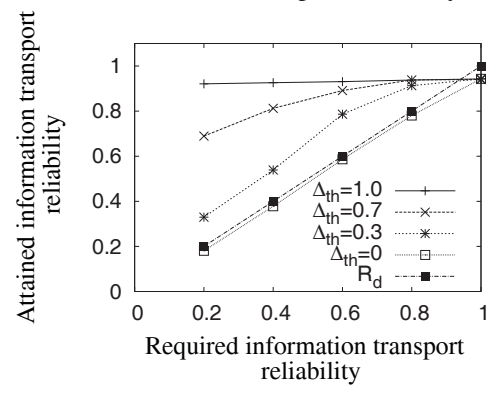

(d) Impact of $\Delta_{t h}$

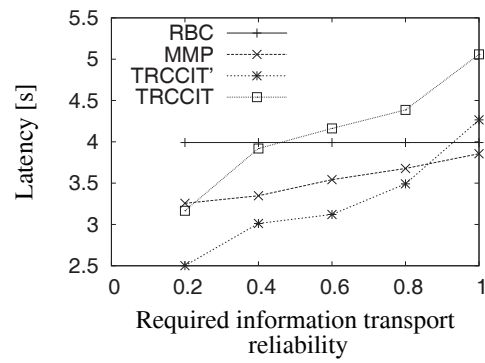

(c) Information transport timeliness

Fig. 1. Tunable reliability of information transport

\section{Performance Evaluation: Tunability and Validation}

In order to evaluate our approach we first describe an evaluation scenario followed by the methodology adopted and simulation settings. Next, we define the performance metrics upon which we evaluated our approach. In last we present our simulation results.

\section{A. Evaluation Scenario and Settings}

We consider a scenario where the phenomenon of interest corresponds to spatio-temporal bursts of information. Only the sensor nodes covered by the spatial phenomena generate information and transport along SP towards the sink. This incurs a substantial congestion on the nodes along the path. Before some phenomenon occurs the information flow is very low and the network does not experience congestion.

We evaluate our approach based on simulations that we have conducted using the TOSSIM [35] simulator. TOSSIM is an eventdriven simulation tool widely used in the WSN community. The general simulation settings are summarized in Table I.

\begin{tabular}{|c|c|}
\hline Number of nodes & 100 \\
\hline Node Placement & Grid \\
\hline Area & $60 \times 60 \mathrm{~m}^{2}$ \\
\hline Distance between two nodes & $5 \mathrm{~m}$ \\
\hline Message size & 29 bytes \\
\hline Buffer size & $36 \mathrm{msgs}$ \\
\hline$\alpha$ & 0.1 \\
\hline
\end{tabular}

TABLE I

SIMULATION ENVIRONMENT SETTINGS

Note that we use grid topology in this paper to emulate uniformly dense deployment and is not a requirement for our algorithms. The sink is located at one corner of the area. Four nodes in the opposite corner of the sink generate atomic information and are randomly selected. The average hop distance between the source nodes and sink is 9 hops. Note that the sources in our simulations were placed at the corner to get a sufficient number of hops to the sink. In a large deployment of hundreds of nodes, these sources need not be at the corner of the deployment. Results were recorded when the system reached a steady state. For every simulation run the node generate 50 messages to be transported towards the sink. We have used the empirical radio model [36] provided by TOSSIM. In this model, a sensor node sends and receives messages using an error distribution based on empirical data. For MAC we have used default CSMA-based implementation.

We compared the TRCCIT with TRCCIT', Modified MMSPEED protocol (MMP) and RBC. TRCCIT' is a variant of TRCCIT which exploits only the temporal redundancy, i.e., adaptive retransmissions and does not split information upon congestion. We have compared TRCCIT' to see the advantage of exploiting the spatial redundancy when congestion builds up. Since this paper deals with reliability of information and not with real time delivery, we have compared our approach with reliability of MMSPEED. As the code for MMSPEED is not available for TOSSIM, we implemented MMP which consists of only the reliability module. For fair comparison we used CSMA/CA without RTS/CTS suggested by the MMSPEED. We compared TRCCIT with MMP in order to observe the impact of on-demand and always available multiple paths. We also compared TRCCIT with RBC. Since RBC specifically provides reliable information transport in presence of high information rates. We do not compare our work to approaches that propose to limit rate, throttling of information, or multipaths as the congestion control schemes. Since they either treat all information equal, i.e., no tunability of information transport or assume congestion is long lasting and utilize back pressure schemes to mitigate the congestion. We also do not compare our scheme with [28], since their system model is different and they consider only two levels of reliability.

For underlying routing we modified Logical Grid Routing (LGR) [37] protocol. LGR creates a spanning tree rooted at the sink and periodically exchanges topology information to maintain the spanning tree. We modified it in such a way that along with next hop on the 


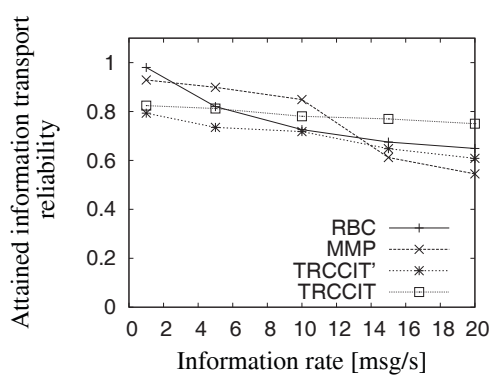

(a) Information transport reliability

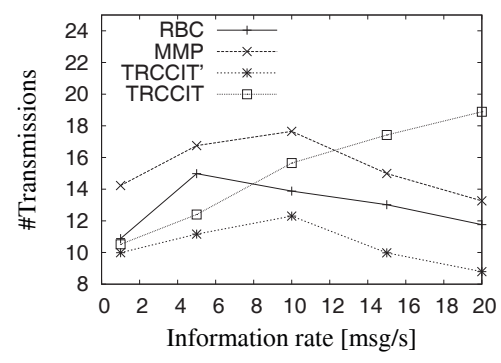

(b) Information transport efficiency

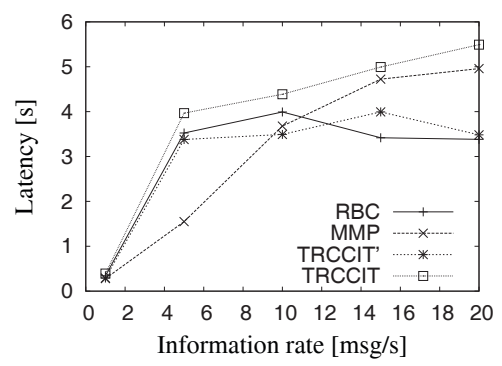

(c) Information transport timeliness

Fig. 2. Adaptation to information rate $\left(R_{d}=0.8\right)$

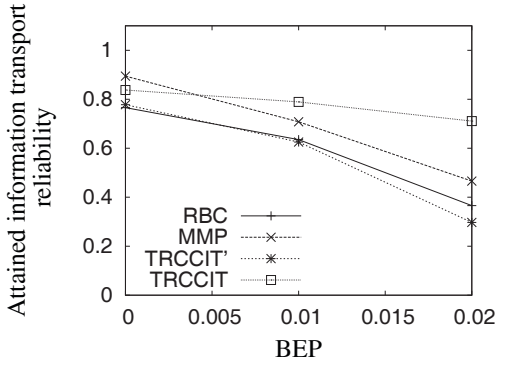

(a) Information transport reliability

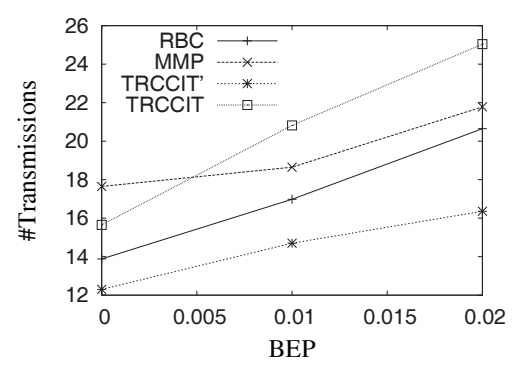

(b) Information transport efficiency

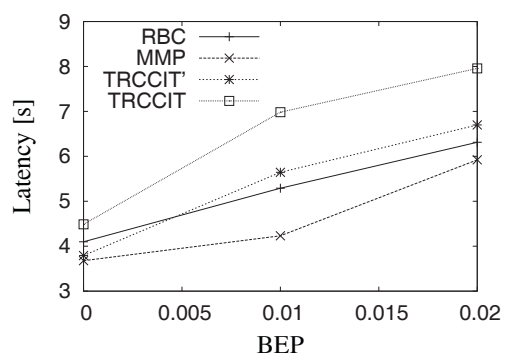

(c) Information transport timeliness

Fig. 3. Adaptation to network conditions $\left(R_{d}=0.8\right)$

spanning tree it also provides the list of neighbors reachable by the node.

\section{B. Performance Metrics}

The performance of TRCCIT is measured in terms of responsiveness and efficiency. The responsiveness is the information transport reliability and timeliness, and the efficiency is mainly given by its message complexity.

Information Transport Reliability: The information transport reliability is the ratio of amount of information received by the sink to the total amount of the information generated.

Timeliness: The timeliness is defined as the time elapsed from the generation of the first information message to the arrival of the first information message at the sink. The timeliness of the protocol is the average of information transport latencies of all generated information. As some information may not be reported at the sink, we do not consider the corresponding messages in the calculation of the average information transport latency.

Efficiency: The efficiency is measured in terms of message complexity. We define the message complexity as the total number of message transmissions required for the information transport (including the retransmissions). We note here that communication between nodes is regarded as the highest energy consuming factor. Therefore, this metric can be utilized to estimate the energy efficiency of the TRCCIT.

\section{Simulation Results}

Now we present our simulation results for different studies we conducted, i.e., impact of tunable reliability, information rates and network conditions.

(1) Tunable Reliability of Information Transport: First we evaluate the performance of TRCCIT for tunable application requirements. In this study the sensor nodes generate the information at 10 messages per second $(\mathrm{msg} / \mathrm{s})$ and transport them towards the sink.
Fig. 1(a) shows the tunability of the different protocols. Since RBC does not provide tunability it achieves a constant reliability, which is less than 1 . Though RBC is developed specifically to cope the bursty nature of information, it can not handle high information rate. MMP also shows almost constant behavior since it always tries to provide higher reliability then required. TRCCIT' on the other hand tries to fulfill tunability requirement but start dropping information along the path when congestion builds up. TRCCIT fulfills the tunable reliability requirements despite the high information rate by dispersing the information on different neighbor nodes. This is also evident from Fig. 1(b) where TRCCIT shows more transmissions compared to TRCCIT'. This behavior validates the impact of Alg. (2) where TRCCIT adapts to high information rate and disperses information to neighbors resulting in higher number of transmissions. MMP and RBC have always higher number of transmissions due to the fact that MMP sends information on multiple paths and RBC upon information loss retransmits. Number of transmissions for TRCCIT remains low for application reliability 0.2 to 0.8 , since it adapts (\#ret), suppresses (Eq. (1)) and utilize HACK (Section IV-B1) with respect to application requirements and network conditions. For reliability 1.0 TRCCIT have higher number of transmissions which corresponds directly to the higher reliability achieved then any other protocol. Fig. 1(c) shows the timeliness tradeoff for different protocols. TRCCIT latency is higher then other protocols which directly correspond to the reliability attained by TRCCIT. The local timer management accurately estimates the retransmission timers such that with less number of transmissions TRCCIT achieve desired reliability. Local timer management shows bit conservative approach but provides tunable reliability with less number of transmissions. For TRCIT' the timeliness remains low, which corresponds to the low reliability attained. MMP shows low latency compared to the TRCCIT due to end to end multiple paths. This can be beneficial in some cases, i.e., real time applications, but at the expense of higher number of transmissions. Fig. 1(d) shows how TRCCIT behaves depending on 
$\Delta_{t h}$. With increasing $\Delta_{t h}$ we observe that the attained reliability is proportionally increased according to desired reliability. This behavior of $\Delta_{t h}$ confirms that the information transport is always bounded between $R_{d}$ and $\left(R_{d}+\Delta_{t h}\right) . \Delta_{t h}=1.0$ leads to the fact that TRCCIT will not suppress the messages and always transport them towards next hop and behaves similar to MMP and RBC, i.e., always provide higher reliability.

(2) Adaptation to Information Rate: As information rate impacts the congestion level, here we observe how the different protocols adapt according to the increasing information rate. In this study we assume that the application requires 0.8 reliability. Fig. 2(a) shows that the TRCCIT adapts to the information rate in an appropriate way then all other protocols. At low information rate, i.e., when there is less congestion RBC and MMP provide higher reliability than required and TRCCIT adapts to provide the required reliability and probabilistically suppress the information. Similarly, TRCCIT' also provides desired reliability at low information rate. But as soon as the information rate is increased MMP, TRCCIT' and RBC degrades whereas TRCCIT maintains the desired application reliability owing to adaptive spatial reuse. Reliability of MMP decreases because of increasing information flow on multiple paths which result in high collisions and dropping of messages. RBC also fails to avoid congestion due to high information rate and start to drop the messages. Fig. 2(b) shows the increase of transmissions for TRCCIT as it adapts to utilize the multiple paths. It is noteworthy to say that the number of transmissions for all other protocols decreases, with the increasing information rate. This is due to the fact that the protocols drop the information due to congestion. Similar effect can be observed for timeliness in Fig. 2(c) where for RBC, MMP and TRCCIT' latency decreases which is directly proportional to reliability and dropped information. Whereas TRCCIT follows split paths and local timer management resulting in high latency. It is also interesting to observe that the latency of MMP increases with high information rate. MMP utilizes more paths due to less reliability across hops (more collisions) which results in longer paths.

(3) Adaptation to Network Conditions: Finally, we observe the effect of different network conditions. In this study the information rate is $10 \mathrm{msg} / \mathrm{s}$ and the application require 0.8 information transport reliability. Fig. 3(a) shows TRCCIT effectively adapts to perturbations due to the fact that TRCCIT utilizes the adaptive retransmissions and splits the information to multiple neighbors upon congestion. On the other hand RBC utilizes fixed number of retransmissions and drops the information once congestion is encountered. Similarly, TRCCIT' and MMP also drops the messages and does not provide the required information transport reliability as BEP increases. Fig. 3(b)-(c) confirms the behavior with growing number of transmissions and timeliness for TRCCIT. We also observe that at BEP 0.0 TRCCIT' performs better than MMP and RBC with respect to number of transmission and latency, since it adapts to the application requirements. At higher BEP the number of transmissions increases due to higher number of retransmissions resulting in higher latency.

Overall our simulations show that TRCCIT outperforms RBC, MMP and TRCCIT' with respect to providing tunable reliability and efficiently mitigating the congestion.

\section{Vi. CONCLUSion \& Future Work}

In this paper, we have proposed TRCCIT, a tunable reliability with congestion control for information transport in WSNs. TRCCIT provides desired application reliability despite evolving network conditions by adaptive retransmissions and suppressing the unnecessary information. Reliability of information transport is achieved by
HACK mechanism aided by localized retransmission timer management. TRCCIT efficiently monitors the information flow and adapts between single path and multiple paths in order to alleviate congestion such that desired application reliability is maintained. In future, we will explore how to enhance TRCCIT to overcome long lasting congestion inside the network and deploy it on real testbed.

\section{ACKNOWLEDGMENT}

This research is supported in part by MUET, HEC, EU CoMiFin, EU INSPIRE, DFG GRK 1362 (TUD GK MM), Think-Trust and Inco-Trust.

\section{REFERENCES}

[1] I. F. Akyildiz, W. Su, Y. Sankarasubramaniam, and E. Cayirci, "Wireless sensor networks: a survey," Computer Networks, vol. 38, no. 4, pp. 393 422, 2002.

[2] F. Stann and J. Heidemann, "Rmst: Reliable data transport in sensor networks," in Proceedings of the First International Workshop on Sensor Net Protocols and Applications, 2003, pp. 102-112.

[3] S. Kim, R. Fonseca, and D. Culler, "Reliable transfer on wireless sensor networks," in IEEE Sensor and Ad Hoc Communications and Networks (SECON), 2004, pp. 449-459.

[4] Y. Sankarasubramaniam, Ö. B. Akan, and I. F. Akyildiz, "Esrt: eventto-sink reliable transport in wireless sensor networks." in Interational Symposium on Mobile Ad Hoc Networking and Computing (MobiHoc), 2003, pp. 177-188.

[5] D. Ganesan, R. Govindan, S. Shenker, and D. Estrin, "Highly-resilient, energy-efficient multipath routing in wireless sensor networks," SIGMOBILE Mob. Comput. Commun. Rev., vol. 5, no. 4, pp. 11-25, 2001.

[6] H. Zhang, A. Arora, Y. Choi, and M. G. Gouda, "Reliable bursty convergecast in wireless sensor networks," in Interational Symposium on Mobile Ad Hoc Networking and Computing (MobiHoc), 2005, pp. 266-276.

[7] F. K. Shaikh, A. Khelil, and N. Suri, "A comparative study for data transport protocols for wsn," in IEEE International Symposium on a World of Wireless, Mobile and Multimedia Networks (WOWMOM), 2008, pp. $1-9$.

[8] B. Hull, K. Jamieson, , and H. Balakrishnan, "Mitigating congestion in wireless sensor networks," in Proc. of ACM SenSys, 2004, pp. $134-$ 147.

[9] C.-Y. Wan, S. B. Eisenman, and A. T. Campbell, "Coda: Congestion detection and avoidance in sensor networks," in Proceedings of the International Conference on Embedded Networked Sensor Systems, 2003, pp. 266-279.

[10] T. He, F. Ren, C. Lin, and S. Das, "Alleviating congestion using trafficaware dynamic routing in wireless sensor networks," in Conference on Sensor, Mesh and Ad Hoc Communications and Networks (SECON), 2008, pp. 233-241.

[11] K. Jaewon, Z. Yanyong, and B. Nath, "Tara: Topology-aware resource adaptation to alleviate congestion in sensor networks," IEEE Transactions on Parallel and Distributed Systems, vol. 18, no. 7, pp. 919-931, 2007.

[12] L. Popa, C. Raiciu, I. Stoica, and D. Rosenblum, "Reducing congestion effects in wireless networks by multipath routing," in Proceedings of the IEEE International Conference on Network Protocols (ICNP), 2006, pp. 96-105.

[13] F. K. Shaikh, A. Khelil, and N. Suri, "AReIT: Adaptable reliable information transport for service availability in wireless sensor networks," in In Proc. of International Conference on Wireless Networks (ICWN), 2009, pp. 75-81.

[14] B. Deb, S. Bhatnagar, and B. Nath, "Information assurance in sensor networks," in In Proceedings of the ACM Conference on Wireless Sensor Networks and Applications (WSNA), 2003, pp. 160-168.

[15] N. Tezcan and W. Wang, "Art: an asymmetric and reliable transport mechanism for wireless sensor networks," Int. J. Sen. Netw., vol. 2, no. 3/4, pp. 188-200, 2007.

[16] B. Marchi, A. Grilo, and M. S. Nunes, "Dtsn: Distributed transport for sensor networks," in Proceedings of the 12th IEEE Symposium on Computers and Communications (ISCC), 2007, pp. 165-172. 
[17] Y. G. Iyer, S. Gandham, and S. Venkatesan, "Stcp: A generic transport layer protocol for wireless sensor networks," in International Conference on Computer Communications and Networks (ICCCN), 2005, pp. 449 454.

[18] E. Felemban, C.-G. Lee, and E. Ekici, "Mmspeed: Multipath multi-speed protocol for qos guarantee of reliability and timeliness in wireless sensor networks," IEEE Transactions on Mobile Computing, vol. 5, no. 6, pp. 738-754, 2006.

[19] B. Deb, S. Bhatnagar, and B. Nath, "Reinform: Reliable information forwarding using multiple paths in sensor networks," in Proceedings of the 28th Annual IEEE International Conference on Local Computer Networks (LCN), 2003, pp. 406-415.

[20] J. Kulik, W. Heinzelman, and H. Balakrishnan, "Negotiationbased protocols for disseminating information in wireless sensor networks," in ACM MobiCom (MobiCom), 1999.

[21] S.-J. Lee and M. Gerla, "Split multipath routing with maximally disjoint paths in ad hoc networks," in International Conference on Communications (ICC), 2001.

[22] J.-Y. Teo, Y. Ha, and C.-K. Tham, "Interference-minimized multipath routing with congestion control in wireless sensor network for high-rate streaming," IEEE Transactions on Mobile Computing, vol. 7, no. 9, pp. 1124-1137, Sept 2008.

[23] C.-W. Hsu, C.-S. Shieh, and W. K. Lai, "A multi-path routing protocol with reduced control messages for wireless sensor networks," in International Conference on Intelligent Information Hiding and Multimedia Signal Processing (IIH-MSP), 2007, pp. 671-675.

[24] C. Wang, B. Li, K. Sohraby, M. Daneshmand, and Y. Hu, "Upstream congestion control in wireless sensor networks through cross-layer optimization," IEEE Journal on Selected Areas in Communications, vol. 25, no. 4, pp. 786-798, 2007.

[25] S. Rangwala, R. Gummadi, R. Govindan, and K. Psounis, "Interferenceaware fair rate control in wireless sensor networks," SIGCOMM Comput. Commun. Rev., vol. 36, no. 4, pp. 63-74, 2006.

[26] S. Chen and N. Yang, "Congestion avoidance based on lightweight buffer management in sensor networks," IEEE Trans. Parallel Distrib. Syst., vol. 17, no. 9, pp. 934-946, 2006.

[27] J. Paek and R. Govindan, "Rcrt: rate-controlled reliable transport for wireless sensor networks," in SenSys, 2007, pp. 305-319.
[28] R. Kumar, R. Crepaldi, H. Rowaihy, A. F. Harris, G. Cao, M. Zorzi, and T. F. L. Porta, "Mitigating performance degradation in congested sensor networks," IEEE Transactions on Mobile Computing, vol. 7, no. 6, pp. 682-697, 2008.

[29] C. Wang, M. Daneshmand, B. Li, and K. Sohraby, "A survey of transport protocols for wireless sensor networks," IEEE Network Magazine, Special Issue on Wireless Sensor Networking, vol. 20, no. 3, pp. 34-40, 2006.

[30] M. A. Rahman, A. E. Saddik, and W. Gueaieb, "Wireless sensor network transport layer: State of the art," in Sensors: Advancement In Modeling, Design Issues, Fabrication And Practical Applications, ser. LECTURE NOTES IN ELECTRICAL ENGINEERING, S. Mukhopadhyay and R. Huang, Eds., vol. 21. Springer-Verlag, 2008, pp. 221-245.

[31] A. Arora, P. Dutta, S. Bapat, and V. K. et al., "A line in the sand: a wireless sensor network for target detection, classification, and tracking." Computer Networks, vol. 46, no. 5, pp. 605-634, 2004.

[32] G.-S. Ahn, S. G. Hong, E. Miluzzo, A. T. Campbell, and F. Cuomo, "Funneling-mac: a localized, sink-oriented mac for boosting fidelity in sensor networks," in Proceedings of the 4th international conference on Embedded networked sensor systems (SenSys), 2006, pp. 293-306.

[33] S. Park, R. Vedantham, R. Sivakumar, and I. F. Akyildiz, "A scalable approach for reliable downstream data delivery in wireless sensor networks." in Proceedings of the Interational Symposium on Mobile Ad Hoc Networking and Computing (MobiHoc), 2004, pp. 78-89.

[34] C. Wan, A. T. Campbell, and L. Krishnamurthy, "Psfq: a reliable transport protocol for wireless sensor networks." in International Workshop on Wireless Sensor Networks and Applications (WSNA), 2002, pp. 1-11.

[35] P. Levis, N. Lee, M. Welsh, and D. Culler, "Tossim: accurate and scalable simulation of entire tinyos applications," in Proceedings of the International Conference on Embedded Networked Sensor Systems (SenSys), 2003, pp. 126-137.

[36] A. Woo, T. Tong, and D. Culler, "Taming the underlying challenges of reliable multihop routing in sensor networks," in Proceedings of the International Conference on Embedded Networked Sensor Systems (SenSys), 2003, pp. 14-27.

[37] Y. Choi, M. G. Gouda, H. Zhang, and A. Arora, "Stabilization of grid routing in sensor networks," Journal of Aerospace Computing, Information and Communication, vol. 3, pp. 214-233, 2006. 